\title{
Antidepresivos inhibidores de la recaptacion de serotonina y sangrado gastrointestinal alto en pacientes ancianos
}

Inhibition of serotonin reuptake by antidepressants and upper gastrointestinal bleeding in elderly patients. Retrospective cohort study. Van Walraven C, Mamdani M M, Wells P S y col. BMJ.2001, 323655

\section{Objetivo}

Determinar la asociación entre los antidepresivos inhibidores de la recaptación de la serotonina (IRS) y el sangrado gastrointestinal alto.

\section{Diseño}

Estudio poblacional retrospectivo de cohortes.

\section{Lugar}

Canada, Ontario.

\section{Participantes}

Se incluyeron 317.824 sujetos mayores de 65 años seguidos por más de 130.000 personas/año.Los sujetos comenzaron a tomar el antidepresivo entre 1992 y 1998. Fueron agrupados según el grado de inhibicion de la recaptación de serotonina de la droga que ingerían. El período de observación se realizó hasta que los pacientes suspendieron la droga, presentaron un sangrado gastrointestinal alto, murieron o finalizó el estudio.

\section{Evaluacion de los factores de riesgo}

Se controló por edad y el sexo (factores asociados a sangrados gastrointestinales altos) y se consideraron antecedentes de sangrados gastrointestinales previos y diabetes. Se clasificó a los pacientes con sangrado gastrointestinal si al egreso hospitalario la base de datos indicaba que habían sido admitidos con el diagnóstico primario de sangrado gastrointestinal alto. Se recabó información sobre otras drogas como los anti-inflamatorios no esteroideos, glucocor ticoides, anticoagulantes, bloqueantes h2 e inhibidores de la bomba de protones.Se definieron los siguientes grupos según su grado de inhibición de la recaptación de sero- tonina: Alta: Paroxetina, Clomipramina, Sertralina y Fluoxetina; Intermedio: Imipramina, Amitriptilina, Venlafaxina; Bajo: desipramina, Nor triptilina, Trazodiona, Bupropion.

\section{Medición de resultados principales}

Admisión hospitalaria por sangrado gastrointestinal alto.

\section{Resultados principales}

En total fueron detectados 974 sangrados, con una tasa de sangrados totales de 7.3 por 1000 personas/año.

Despues de controlar por edad, sexo, drogas y sangrado gastrointestinal previo, el riesgo de sangrado se incrementó significativamente en un $10.7 \%$ y $9.8 \%$ respectivamente al aumentar la inhibición de la recaptación de serotonina. Diferencias absolutas en el sangrado entre los grupos de antidepresivos fueron mayores para los octogenarios (baja inhibición de la recaptación de la serotonina, 10.6 sangrados cada 1000 personas/ año vs alta inhibición de la recaptación de la serotonina, 14.7 sangrados cada 1000 personas/ año.

En los individuos con antecedentes de sangrado gastrointestinal alto previo el grupo de baja inhibicion tuvo 28.6 sangrados cada 1000 personas/ año vs los de alta inhibición, 40,3 sangrados cada 1000 personas/ año.

\section{Conclusión}

Luego de controlar según edad, sexo o sangrado gastrointestinal alto previo, los antidepresivos con alta inhibición de la recaptación de serotonina incrementaron el riesgo de sangrado gastrointestinal alto.Dicho incremento fue clínicamente importante para los ancianos y aquellos con antecedentes de sangrado gastrointestinal.

\section{Comentario}

El sindrome depresivo en los ancianos se observa frecuentemente en la práctica clínica ambulatoria. Es un diagnóstico detectado y tratado frecuentemente por el médico de atención primaria 1. Existe actualmente una tendencia a utilizar medicamentos del grupo de los inhibidores de la recaptación de la serotonina como droga de primera elección, en reemplazo de los antidepresivos tricíclicos dados sus "menores efectos adversos" 2. Hasta la aparición de este trabajo solo existían estudios caso control donde se observaba mayor riesgo de sangrado gastrointestinal alto con la ingesta de inhibidores de la recaptación de serotonina.La validez de estos estudios fue cuestionada ya que los antidepresivos no fueron especificamente clasificados por su capacidad de inhibición de la recaptación de la serotonina.
Este estudio nos aporta conocimiento sobre un efecto adverso no tan conocido, de gran importancia clínica para los pacientes con alto riesgo de sangrado como son los octogenarios y aquellos con sangrado digestivo alto previo.Los antidepresivos del grupo de los inhibidores de la recaptación de la serotonina tienen, como todos las drogas, sus indicaciones sugeridas, efectos adversos y contraindicaciones precisas que es importante conocer al momento de prescribirlos. A la luz de estos nuevos hallazgos, quizas sea conveniente rever las indicaciones de los "antidepresivos antiguos", con precios mas accesibles, e indicarlos correctamente, como así también, restringir el uso de los inhibidores de la recaptación de la serotonina en pacientes con alto riesgo de sangrado gastrointestinal. 